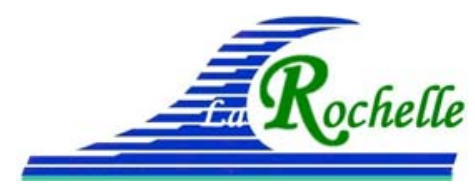

XVèmes Journées Nationales Génie Côtier - Génie Civil

La Rochelle, 29 au 31 mai 2018

DOI:10.5150/jngcgc.2018.002 (C) Editions Paralia CFL

disponible en ligne - http://www.paralia.fr - available online

\title{
Transferts d'énergie et réflexion des ondes infra-gravitaires au niveau d'une plage dissipative
}

\author{
Xavier BERTIN ${ }^{1}$, Anouk de BAKKER ${ }^{1}$, Thomas GUERIN ${ }^{1}$, \\ Teddy CHATAIGNER ${ }^{1}$, Kévin MARTINS ${ }^{1}$, Thibault COULOMBIER ${ }^{1}$
}

\author{
1. UMR 7266 LIENSs, CNRS-Université de La Rochelle, 2 rue Olympe de Gouges, \\ 17000 La Rochelle, France. \\ xbertin@univ-lr.fr
}

\section{Résumé :}

Les ondes infra-gravitaires (IG) sont des ondes de surface avec des périodes de l'ordre de 25 à $250 \mathrm{~s}$ liées à la présence de groupes dans les vagues incidentes. Lors d'épisodes de forte houle, les ondes IG ont une contribution essentielle dans la dynamique hydrosédimentaire des zones littorales, particulièrement au niveau des plages dissipatives. Notre étude repose sur une campagne de mesures inédite, réalisée au niveau de la plage dissipative de Saint-Trojan (SO de l'Ile d'Oléron) pendant la tempête Kurt (03 février 2017), caractérisée par des vagues incidentes au large de hauteur significative supérieure à $9 \mathrm{~m}$ et de périodes de pic atteignant $22 \mathrm{~s}$. Les données expérimentales sont complétées par l'application du système de modélisation XBeach, afin de mieux comprendre les mécanismes de génération et les transformations des ondes IG en eau peu profonde. L'analyse des données expérimentales révèle la présence d'ondes IG exceptionnelles, dont la hauteur significative atteint $1.85 \mathrm{~m}$. XBeach permet de reproduire le développement de telles ondes IG et l'analyse des résultats numériques révèle que leur développement est amplifié par la présence d'un déphasage avec l'enveloppe de l'énergie des vagues incidentes. L'analyse spectrale de la surface libre mesurée et modélisée montre le développement d'ondes IG super-harmoniques, dont l'énergie est dissipée en eau peu profonde et d'ondes IG subharmoniques, qui dominent le contenu spectral de la surface libre le long du rivage. L'analyse des données expérimentales montre une forte modulation tidale de la réflexion des ondes IG, avec une réflexion presque totale à marée haute $\left(\mathrm{R}^{2} \sim 1.0\right)$ et une réflexion plus faible à marée basse $\left(\mathrm{R}^{2} \sim 0.3-0.5\right)$. Cette modulation tidale est expliquée par l'augmentation de la pente $\mathrm{du}$ fond vers le haut de plage. Les coefficients de réflexion obtenus restent cependant très élevés pour une plage dissipative, ce que nous expliquons par le développement d'ondes IG subharmoniques de période de l'ordre de $300 \mathrm{~s}$, qui subissent très peu de dissipation.

\section{Mots-clés :}

Ondes infra-gravitaires, Plage dissipative, Transferts d'énergie, Réflexion, Dissipation, Ile d'Oléron. 


\section{Thème 1 - Hydrodynamique côtière}

\section{Introduction}

Les ondes infragravitaires (IG) correspondent à des ondes de gravité se propageant à la surface des océans avec des périodes de l'ordre de 25 à $250 \mathrm{~s}$ et sont liées à la présence de groupes dans les vagues incidentes. Il est désormais bien admis que les ondes IG ont une contribution essentielle dans l'hydrodynamique littorale (e.g. GUZA et THORNTON, 1982; ELGAR et al., 1992), le transport sédimentaire (e.g. RUSSELL, 1993) ou la rupture des dunes ou des barrières littorales (e.g. ROELVINK et al., 2009). $\mathrm{Au}$ niveau des plages dissipatives, plusieurs études ont montré que les ondes IG dominaient généralement le spectre de l'élévation de la surface libre le long du rivage (e.g. GUZA et THORNTON, 1982; RUSSELL, 1993; RUESSINK et al., 1998). De ce fait, les ondes IG peuvent contrôler le franchissement par paquet de mer au niveau des zones basses du littoral et la mise en place de dépôts de washover, comme c'est le cas au sud de la zone d'étude (BAUMANN et al., 2017). Dans un article de synthèse récent sur les ondes IG, BERTIN et al., (2018) ont cependant montré que les connaissances concernant plusieurs mécanismes associés aux ondes IG restaient rudimentaires, comme par exemple le développement d'un déphasage entre l'onde liée (bound wave) et l'enveloppe de l'énergie des vagues, la génération d'harmoniques ou encore la dissipation et la réflexion des ondes IG le long du rivage. Afin d'améliorer ces connaissances, nous présentons une étude qui repose sur une campagne de mesures inédite, réalisée au niveau de la plage dissipative de Saint-Trojan (SO de l'Ile d'Oléron) pendant la tempête Kurt (03 février 2017). Les données expérimentales sont complétées par l'application du système de modélisation XBeach, afin de mieux comprendre les mécanismes de génération et les transformations des ondes IG en eau peu profonde.

\section{Description de la zone d'étude}

La plage de Saint-Trojan est située au SO de l'Ile d'Oléron (figure 1), qui correspond à une flèche sableuse longue de $8 \mathrm{~km}$, limitée au sud par l'embouchure tidale de Maumusson. La zone pré-littorale en face de la plage étudiée présente une pente très faible, l'isobathe $20 \mathrm{~m}$ étant située à environ $10 \mathrm{~km}$ du trait de côte. Le régime de marée est macro-tidal et semi-diurne, avec un marnage atteignant $5.5 \mathrm{~m}$ en vives-eaux. D'après BERTIN et al. (2008), le régime de vagues en eau profonde au large de la zone d'étude est dominé par des vagues de hauteur significative $\left(\mathrm{H}_{\mathrm{s}}\right)$ de l'ordre de $2.0 \mathrm{~m}$, des périodes de pic $\left(\mathrm{T}_{p}\right)$ de 8 à $12 \mathrm{~s}$ et une direction moyenne d'ouest à ouest-nord-ouest. Lors des tempêtes, les $\mathrm{H}_{\mathrm{s}}$ au large peuvent atteindre voire dépasser $10 \mathrm{~m}$ avec des $\mathrm{T}_{\mathrm{p}}$ supérieures à $20 \mathrm{~s}$ (BERTIN et al., 2015). Cette plage est composée de sables fins $\left(\mathrm{d}_{50} \sim 0.2 \mathrm{~mm}\right)$ et présente une morphologie dissipative, avec des pentes de l'ordre de 1/80. Après la persistance de vagues de faible énergie, des systèmes de barres intertidales de faible amplitude ainsi qu'une berme de pente modérée (1/25) peuvent se développer (BERTIN et al., 2008). 


\section{XVèmes Journées Nationales Génie Côtier - Génie Civil \\ La Rochelle, 29 au 31 mai 2018}

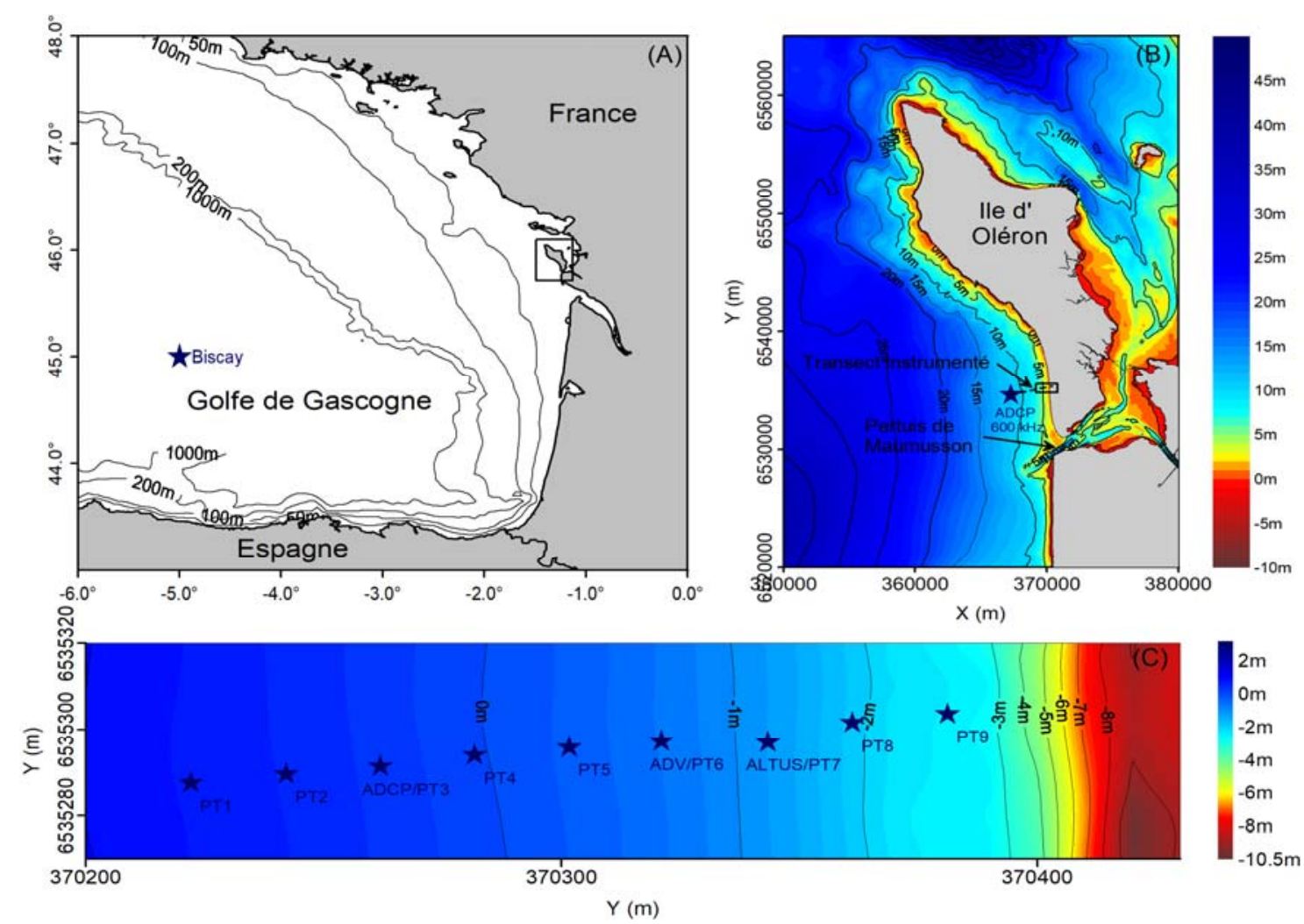

Figure 1. (A) Bathymétrie du Golfe de Gascogne et localisation de la zone d'étude, (B) bathymétrie de l'Ile d'Oléron et localisation du transect instrumenté et (C) bathymétrietopographie de la zone intertidale et position des instruments déployés.

\section{Méthodes et données}

\subsection{Description de la campagne de mesure}

Une campagne de mesure a été réalisée entre le 30/01/2017 et le 09/02/2017 pendant la tempête Kurt $(03 / 02 / 2017)$, qui a généré des vagues $H_{s}=9.5 \mathrm{~m}$ et $T_{p}=22 \mathrm{~s}$ au niveau de la bouée Biscay (figure 1-A). Un courantomètre ADCP équipé d'un capteur de pression a été déployé par $10 \mathrm{~m}$ de fond au large de la plage étudiée (figure 1-B). 9 capteurs de pression, un courantomètre ADCP et un courantomètre ADV ont été déployés tous les $20 \mathrm{~m}$ le long d'un transect perpendiculaire à la plage du 01/02/2017 au 03/02/2017. La topographie de la plage intertidale a été mesurée au GPS différentiel à chaque marée basse et les évolutions morphologiques observées se sont avérées très faibles (i.e. $\Delta \mathrm{z}<0.1 \mathrm{~m}$ ).

\subsection{Traitement des données expérimentales}

Les mesures de pressions au fond ont été corrigées de la pression atmosphérique mesurée au nord de l'Ile d'Oléron (figure 1-B) et des échantillons de 30 minutes ont été analysés (seuls ceux où la hauteur d'eau minimale excède $0.1 \mathrm{~m}$ ont été considérés). Des 


\section{Thème 1 - Hydrodynamique côtière}

spectres de densité d'énergie de la pression au fond $E_{\mathrm{p}}(\mathrm{f})$ ont été calculés à l'aide de transformées de Fourrier, en utilisant 19 sous-blocs avec un fenêtrage de Hanning (38 degrés de liberté). Ces spectres de pression ont été transformés en spectres d'élévation de la surface libre $\mathrm{E}(\mathrm{f})$ en appliquant une fonction de transfert dérivée de la théorie linéaire des vagues. La hauteur spectrale $\mathrm{H}_{\mathrm{m} 0}$ a été calculée comme suit :

$H_{m 0}=4 \sqrt{m_{0}}$

Où mo est le moment d'ordre 0 défini par :

$m_{0}=\int_{f \text { min }}^{f \max } E(f) d f$

Pour la hauteur $\mathrm{H}_{\mathrm{m} 0}$ dans la bande gravitaire (notée $\mathrm{H}_{\mathrm{m} 0, \mathrm{G}}$ ), $\mathrm{f}_{\max }$ est fixé à $0.4 \mathrm{~Hz}$ et $\mathrm{f}_{\min }$ correspond à la fréquence de coupure entre les bandes gravitaire et IG, qui est adaptative et définie comme la moitié de la fréquence de pic des vagues incidentes, en accord avec HAMM \& PERONNARD (1997).

\subsection{Implémentation du système de modélisation XBeach}

Ces données expérimentales sont complétées par des simulations numériques réalisées à l'aide du système de modélisation XBeach (ROELVINK et al., 2009). XBeach est un système de modélisation 2DH, qui réalise le couplage entre un modèle de Saint-Venant, un modèle spectral de vagues simplifié et un modèle de transport sédimentaire et d'évolution du fond. La représentation des groupes de vagues dans le modèle spectral et le couplage avec le modèle de Saint-Venant à travers les gradients de tension de radiation des vagues permettent de reproduire la génération et la propagation des ondes IG. L'espace géographique est discrétisé selon une grille rectilinéaire s'étendant sur 5 $\mathrm{km} \mathrm{du}$ nord au sud et entre le trait de côte jusqu'à des profondeurs de $27 \mathrm{~m}$ dans la direction est-ouest, avec un pas en espace variant de $20 \mathrm{~m}$ au large à $5 \mathrm{~m}$ dans la zone intertidale. XBeach est forcé avec des séries temporelles de hauteurs d'eau et de spectres directionnels interpolés à partir des modèles régionaux décrits dans BERTIN et al. (2015).

\section{Résultats et discussion}

\subsection{Caractérisation des ondes IG}

L'analyse des données expérimentales montre que les vagues au point de déferlement sont caractérisées par des $\mathrm{H}_{\mathrm{m} 0, \mathrm{G}}$ de $2.0 \mathrm{~m}$ et des $\mathrm{T}_{\mathrm{p}}$ de l'ordre de 13 à $14 \mathrm{~s}$ au début de la campagne et ont atteint $6.0 \mathrm{~m}$ et $22 \mathrm{~s}$ lors de la tempête Kurt (GUERIN et al., 2018). La hauteur des ondes $\mathrm{IG} \mathrm{H}_{\mathrm{m} 0, \mathrm{IG}}$ augmente vers le rivage et atteint un maximum à $1.85 \mathrm{~m}$ au niveau du capteur PT6 (figure 1-C) pendant la tempête Kurt, où elle dépasse $\mathrm{H}_{\mathrm{m} 0, \mathrm{G}}$ (figure 2). Ces évolutions sont raisonnablement reproduites par XBeach, avec des

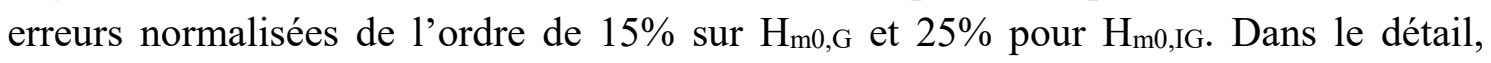




\section{XVèmes Journées Nationales Génie Côtier - Génie Civil La Rochelle, 29 au 31 mai 2018}

XBeach tend à sous-estimer $\mathrm{H}_{\mathrm{m} 0, \mathrm{IG}}$ au niveau du second cycle de marée, où les vagues incidentes étaient caractérisées par un spectre plus large. Cette sous-estimation pour de tels états de mer est cependant un problème connu d'XBeach (ROELVINK et al., 2018).
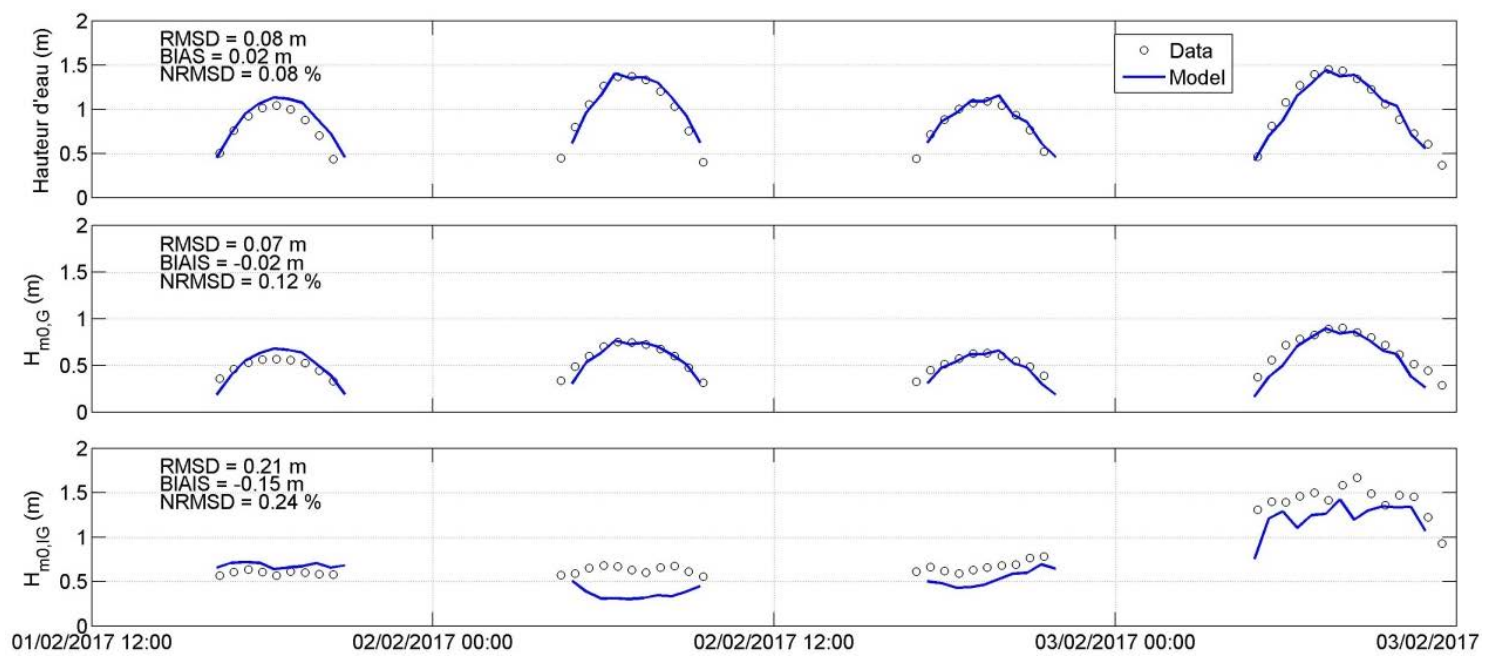

Figure 2. Comparaison entre les hauteurs d'eau (haut), $H_{m 0, G}$ (centre) et $H_{m 0, I G}$ (bas) simulées (courbe bleu) et mesurées (cercles noirs) au niveau du capteur 6 (figure 1-C).

\subsection{Mécanismes de génération}

La hauteur des ondes IG observées lors de notre campagne de mesure correspond aux plus fortes valeurs que nous avons trouvées dans la littérature. D'après la solution analytique de HASSELMANN (1962) pour le calcul de l'onde IG liée, les transferts d'énergie depuis la bande gravitaire vers la bande IG sont maximums lorsque l'énergie incidente des vagues est élevée et distribuée sur un spectre étroit. Dès lors, les vagues incidentes très énergétiques et longues associées à la tempête Kurt expliquent directement le développement de fortes ondes IG. Cependant, des études menées pour des conditions de vagues incidentes comparables (e.g. FIEDLER et al., 2015) montrent le développement d'ondes IG de l'ordre d'1 m, suggérant que d'autres paramètres interviennent à St Trojan. Lorsque la hauteur d'eau diminue, l'onde IG n'est plus exactement en opposition de phase avec les groupes de vagues comme considéré par HASSELMANN (1962) et un déphasage de l'ordre de quelques secondes se développe, augmentant le transfert d'énergie depuis la bande gravitaire vers la bande IG (BERTIN et al., 2018). Bien que ce phénomène ait lieu pour n'importe quel type de plage, nous proposons que son effet soit plus important à St Trojan en étant actifs sur une distance très élevée, du fait de la faible pente de la zone pré-littorale. Cette hypothèse est corroborée par l'analyse des résultats d'XBeach, qui montre qu'un déphasage de d'ordre de quelques secondes est déjà présent à plus d'un km du rivage. 


\section{Thème 1 - Hydrodynamique côtière}

\subsection{Transferts d'énergie}

Afin d'étudier l'évolution de la répartition fréquentielle des ondes IG avec une résolution spectrale suffisante, nous avons analysé des échantillons de 2 heures de l'élévation de la surface libre, centrés sur chaque marée haute. La figure 3 correspond à la répartition spatio-fréquentielle de l'énergie associée aux ondes IG lors de la tempête Kurt, calculée à partir des observations (figure 3-A) et du modèle (figure 3-B). Cette répartition montre des similitudes entre les observations et le modèle, avec en bas de plage un pic principal autour de $0.015 \mathrm{~Hz}$, un pic super-harmonique à $0.03 \mathrm{~Hz}$ et un pic subharmonique avec des basses fréquences de 0.001 à $0.005 \mathrm{~Hz}$. En remontant vers le haut de plage, ces trois pics d'énergie apparaissent à des fréquences plus hautes, et le dernier domine complètement le spectre le long du rivage. Nous proposons que ce phénomène résulte de l'interaction entre ondes incidentes et réfléchies, qui génère des nœuds et anti-nœuds d'ondes quasi-stationnaires, dont la position relative au trait de côte varie en fonction de la fréquence de l'onde et de la pente du fond. Un phénomène similaire a déjà été observé en laboratoire par BATTJES et al. (2004) ou pour les vagues par MARTINS et al. (2017).
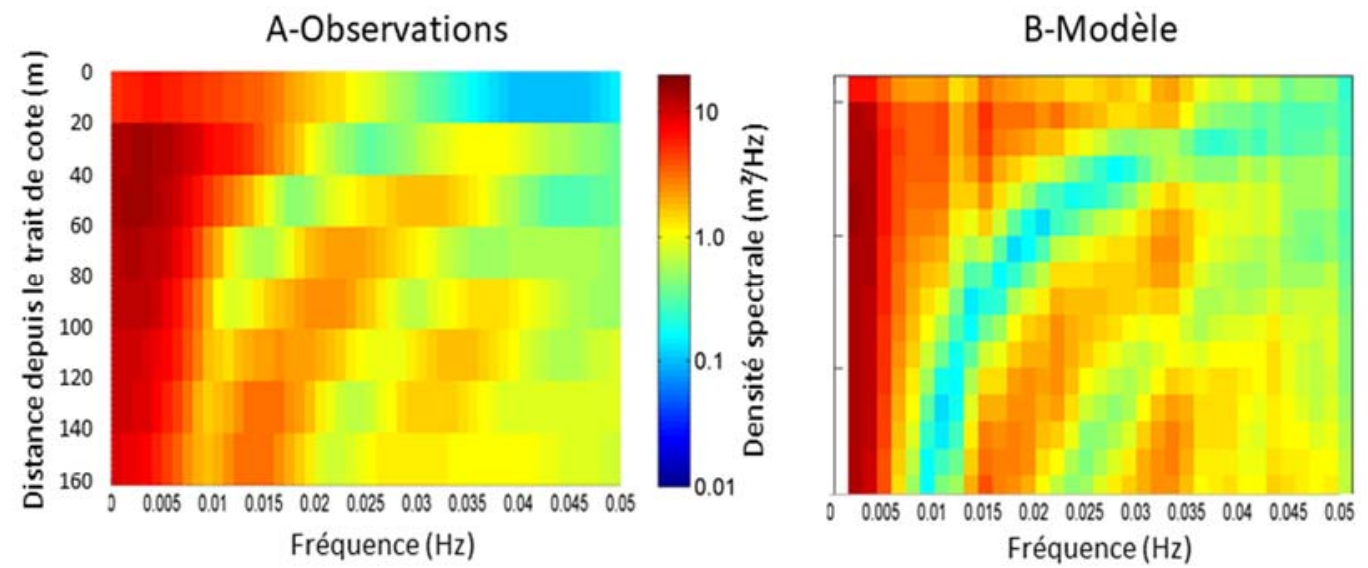

Figure 3. Répartition spatio-fréquentielle de la densité spectrale des ondes IG pendant la tempête Kurt à partir des observations (A) et du modèle (B).

Plusieurs études ont déjà montré que les ondes IG pouvaient générer des superharmoniques au niveau des plages dissipatives, en interagissant entre elles et/ou avec le fond (e.g. DE BAKKER, 2015). A l'inverse, la génération d'ondes subharmoniques à très basse fréquence est moins documentée et pourrait être liée à la présence de groupes à l'échelle des ondes IG, qui forceraient le développement d'ondes à très basse fréquence, mais cette hypothèse fait l'objet de vérifications en cours. 


\section{XVèmes Journées Nationales Génie Côtier - Génie Civil \\ La Rochelle, 29 au 31 mai 2018}

\subsection{Réflexion}

Les données de 1'ADCP de la zone intertidale (figure 1) ont été utilisées afin de séparer les ondes IG incidentes et réfléchies avec la méthode de GUZA et al. (1984). Le rapport entre l'énergie des ondes IG réfléchies et incidentes correspond au coefficient de réflexion $\mathrm{R}^{2}$, qui montre une forte modulation tidale, avec des valeurs de l'ordre de 1 à marée haute et de 0.3 à 0.5 à marée basse (figure 4). Cette modulation tidale est expliquée par l'augmentation de la pente de la plage intertidale depuis $1 / 80$ en bas de plage, où les ondes IG les plus courtes sont dissipées par déferlement, à 1/25 en haut de plage, où les ondes IG sont essentiellement réfléchies. Malgré tout, les valeurs de $\mathrm{R}^{2}$ obtenues restent élevées pour une plage dissipative au regard de la littérature (e.g. BERTIN et al., 2018). Nous proposons que cette forte réflexion soit liée au développement d'ondes IG subharmoniques de très basse fréquence, qui sont trop longues pour subir une dissipation importante (BATTJES et al., 2004).

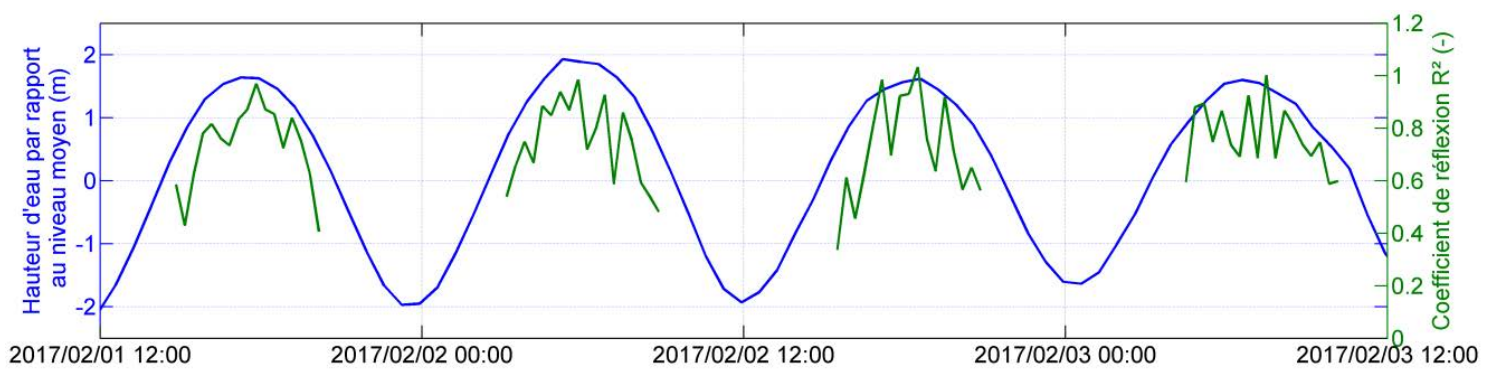

Figure 4. Coefficients de réflexion $R^{2}$ (vert) au niveau du profileur de courant $A D C P$ de la zone intertidale et hauteur d'eau (bleu) au niveau de l'ADCP déployé par $10 \mathrm{~m}$ de fond .

\section{Conclusions et perspectives}

Une campagne de mesure inédite a été réalisée au SO de l'Ile d'Oléron et a montré le développement d'ondes IG exceptionnelles pendant la tempête Kurt. Ces fortes ondes IG sont liées aux vagues incidentes très énergétiques mais aussi à la présence d'une zone pré-littorale en pente très faible, qui permet un transfert d'énergie important depuis la bande gravitaire vers la bande IG. L'analyse spectrale des observations et des résultats numériques montre des transferts d'énergie importants vers des fréquences super-harmoniques et sub-harmoniques en eau peu profondes, qui sont liées à des interactions entre ondes IG. Enfin, les données expérimentales montrent une forte modulation tidale de la réflexion des ondes $\mathrm{IG}$, avec une forte réflexion à marée haute, qui est sans doute liée au développement d'ondes IG à très basses fréquence le long du rivage. Ces interprétations sont en cours de vérification à partir des résultats du modèle. 


\section{Thème 1 - Hydrodynamique côtière}

\section{Remerciements}

Ces travaux de recherche ont été menés dans un programme de Chaire Régionale EVEX. L'ADB est cofinancée par une bourse prestige du programme Campus France. Les équipes qui développent XBeach, sont chaleureusement remerciées.

\section{Références bibliographiques}

BAUMANN J., CHAUMILLON E., BERTIN X., SCHNEIDER J.-L., GUILLOT B., SCHMUTZ M. (2017). Importance of infragravity waves for the generation of washover deposits. Marine Geology, Vol. 391, pp 20-35. https://doi.org/10.1016/j.margeo.2017.07.013

BATTJES J.A., BAKKENES H.J., JANSSEN T.T., VAN DONGEREN A.R. (2004). Shoaling of subharmonic gravity waves. Journal of Geophysical Res. Vol. 109, C02009. https://doi.org/10.1029/2003JC001863

BERTIN X., LI K., ROLAND A., BIDLOT, J.R. (2015). The contribution of short waves in storm surges: two recent examples in the central part of the bay of Biscay. Continental Shelf Research, Vol. 96, pp 1-15. http://dx.doi.org/10.1016/j.csr.2015.01.005

BERTIN X., CASTELlE B., CHAUMILLON E., BUTEL R., QUIQUE R. (2008). Estimation and inter-annual variability of the longshore transport at a high-energy dissipative beach: the St Trojan beach, SW Oléron Island, France. Continental Shelf Research, Vol. 28, pp 1316-1332. https://doi.org/10.1016/j.csr.2008.03.005

BERTIN X., DE BAKKER A., VAN DONGEREN A., COCO G., ANDRÉ G., ARDHUIN F., BONNETON P., BOUCHETTE F., CASTELLE B., CRAWFORD W.C., DAVIDSON M., DEEN M., DODET G., GUÉRIN T., INCH K., LECKLER F., MCCALL R., MULLER H., OLABARRIETA M., ROELVINK D., RUESSINK G., SOUS D., STUTZMANN E., TISSIER M. (2018). Infragravity waves: From driving mechanisms to impacts. Earth-Science Reviews, Vol. 177, pp 774-799.

https://doi.org/10.1016/j.earscirev.2018.01.002

DE BAKKER A.T.M., TISSIER M.F.S., RUESSINK B.G. (2015). Beach steepness effects on nonlinear infragravity-wave interactions: a numerical study. Journal of Geophysical Research, Vol. 121. https://doi.org/10.1002/2015JC011268

ELGAR S., HERBERS T.H.C., OKIHIRO M., OLTMAN-SHAY J., GUZA, R.T. (1992). Observations of infragravity waves. Journal of Geophysical Research, Vol. 97 (C10), 15573-15577. https://doi.org/10.1029/92JC01316

FIEDLER J. W., BRODIE K.L. MCNINCH J. E., GUZA R.T. (2015). Observations of runup and energy flux on a low-slope beach with high-energy, long-period ocean swell. Geophysical Research Letters, Vol. 42, pp 9933-9941. https://doi.org/10.1002/2015gl066124 GUÉRIN T., BERTIN X., COULOMBIER T., DE BAKKER A. (2018). Impacts of wave-induced circulation in the surf zone on wave setup. Ocean Modelling, Vol. 123, pp 86-97. https://doi.org/10.1016/j.ocemod.2018.01.006 


\section{XVèmes Journées Nationales Génie Côtier - Génie Civil La Rochelle, 29 au 31 mai 2018}

GUZA R.T., THORNTON E.B., HOLMAN R.A. (1984). Swash on steep and shallow beaches. Proceedings of the 19th ICCE conference, ASCE, 708-723.

GUZA R.T., THORNTON E.B. (1982). Swash oscillations on a natural beach. Journal of Geophysical Research, Vol. 87, pp 483-491. https://doi.org/10.1029/JC087iC01p00483

HAMM L., PERONNARD C. (1997). Wave parameters in the nearshore: A clarification. Coastal Engineering, Vol. 32, pp 119-135. https://doi.org/10.1016/S03783839(97)81746-2

HASSELMANN K. (1962). On the non-linear transfer in a gravity spectrum, Part 1. General theory. Journal of Fluid Mechanics, Vol. 12, pp 481-500. https://doi.org/10.1017/S0022112062000373

MARTINS K., BLENKINSOPP C.E., ALMAR, R., ZANG J. (2017). The influence of swash-based reflection on surf zone hydrodynamics: a wave-by-wave approach. Coastal Engineering, Vol. 122, pp 27-43. https://doi.org/10.1016/j.coastaleng.2017.01.006

ROELVINK D., RENIERS A., VAN DONGEREN A., VAN THIEL DE VRIES J. , MCCALL R., LESCINSKI J. (2009). Modelling storm impacts on beaches, dunes and barrier islands. Coastal Engineering, Vol. 56, pp 1133-1152. https://doi.org/10.1016/j.coastaleng.2009.08.006

ROELVINK D., MCCALL R., MEHVAR S., NEDERHOFF K., DASTGHEIB A. (2018). Improving predictions of swash dynamics in XBeach: The role of groupiness and incident-band runup. (2018). Coastal Engineering, Vol. 134, pp 103-123. https://doi.org/10.1016/j.coastaleng.2017.07.004

RUESSINK B.G., HOUWMAN K.T., HOEKSTRA P. (1998). The systematic contribution of transporting mechanisms to the cross-shore sediment transport in water depths of 3 to $9 \mathrm{~m}$. Marine Geology, Vol. 152, pp 295-324. https://doi.org/10.1016/S00253227(98)00133-9

RUSSELL P. (1993). Mechanisms for beach erosion during storm. Continental Shelf Research, Vol.13, pp 1243-1265. https://doi.org/10.1016/0278-4343(93)90051-X 
Thème 1 - Hydrodynamique côtière 